\title{
Le rôle du droit comparé dans l'avènement du droit européen
}

\section{Véra Jacob de Fradera}

L'Institut suisse de droit compaté nous offre dans le cadre de ses publications un ouvrage qui nous attire dès la première vue par son intitulé et par la renommée de ses collabotateurs, tous des compatatistes très actifs dans plusieurs domaines de ce champ du droit, soir au milieu universitaire, celui de l'administration bruxellois ou dans les activités propres à la magisttature.

L'ouvrage est le résultat d'un important colloque téalisé à Lausanne du 14 au 15 avril 2000 , sous les auspices de l'Institut suisse de droit comparé, dont le thème central a été une réflexion sur la signification de la méthode comparatiste dans diffétents domaines, et à différents stades de la genèse du droit et dans son application. Les organisateurs de ce colloque voudraient développer des études sur l'étendue de la contribution du droit comparé dans l'élaboration du droit communautaire. Selon les mots de M. Pierre Widmer, dans l' avant-propos de louvrage, celle-ci laisse le lecteur découvrir cette perspective dans toute sa richesse, mais aussi dans son opacité parfois mystérieuse à travers les contributions réunies dans le présent volume $e^{\text {. }}$

1 P. 08 de Pavant-propos. 
L'ouvrage contient six contributions dans plusieurs domaines du droit : le Droit de la consommation (Bernd STAUDER), Droit des societés (Ulrich IMMENGA), Proprieté intelectuelle (André FRANÇON), Protection de la personnalité (Alain BRUN), la perspective du juge de la Cour de Justice des Communautés Européennes (Vassili CHRISTIANOS), Droits de 1 'homme (Paul MAHONEY). La synthèse est présentée par Bernard DUTOIT.

Dans le but de montrer l'influence du droit comparé dans plusieurs secteurs du droit communautaire, l'ouvtage a précisé le cheminement de la démarche comparative à trois niveaux : d'abord, celui du législateur européen, ensuite celui de la Cour de justice des Communautés européennes et en troisième lieu, celui de la Cour européenne des droits de l'Homme.

En ce qui concerne le législateur européen, un des domaines où il a souffert une importante influence du droit comparé est, sans doute, le droit de la consommation, notamment pour l'élaboration des nombreuses directives, dont le but est la protection des consommateurs et la promotion de leurs intérêts. Les directives constituent une remarquable création du droit conmunautaire, et elles visent le rapprochement des législations nationales des pays membres de l'Union européenne dans nombres secteurs du droit prjvé. Le législareur europécn s'est rendu compte du besoin qu'avaient les consommateurs de l'Union européenne de disposer d'un socle minimal commun de règles de droir, capables de renforcer leur. confiance dans les rapports avec les commerçants et les prestataires de service. Le but de ces règles est celui de permettre aux consommateurs une meilleur insertion dans le marché commun. Dans ce sens, on peur nommer comme exemple la Directive 93/13/CE du Conseil du 5 avril 1993, relative aux clauses abusives figurant dans les contrats conclus entre un professionnel er un consommateur, la Directive 97/7/CE du Parlement Européen et du Conseil du 20 mai 1997, sur la protection des consommateurs en matière de contrats à distance entre consommateur er fournisseur, la Directive 1999/44/CE du Parlement Européen et du Conseil du 25 mai 1999, qui porte sur cerrains aspects de la vente et des garanties des biens de consommation er beaucoup d'autres. Par ailleurs, comme a souligné M. Bernd STAUDER dans son excellent rapport ${ }^{3}$, les Directives visent également des problèmes nouveaux, conséquence du développement économique et social (time-share, vente par Internet, e-commerce). C'esr ce mêrne auteur qui affirme que le recours à la méthode comparative devrait être une condition indispensable à l'elaboration des normes harmonisées. Malheureusement, la méthode comparative n'est pas mentionnée dans les considérants, les sources nationales de leur inspiration ne sonr jamais évoquées par les législateurs communautaires. Pour accomplir l'obligation en droit primaire, de recourir à la méthode comparative, le législateur communautaire a choisì la méthode foncrionnelle. Selon cetre

\footnotetext{
${ }^{2}$ Voir à propos la synthèse de colloque par M. Bernard DUTOrT.

${ }^{3}$ In "L'exemple du droit de la consommation", p. 11.
} 
méthode, face à un problène donné, le législateur communautaire récense l'état des législations nationales et met en évidence les convergences et les divergences existantes. L'auteur remarque que l'évaluation de la nécessité d'agir par la voie de l'harmonisation et du champ de lintervention législative est fonction de la compétence des autorités communautaires, donc de l'impact de ces divergences sur le fonctionnement du marché intérieur et sur la protection des consommateurs, et du principe de la subsidiarité. Si la nature du sujet exige l'harmonisation, l'approche fonctionnelle imposerait le choix de la meilleute solution. Comment atteindre la meilleure solution? D'abord, le législateur communautaire peut avoir recours aux solutions nationales, dont il a fait le recensement. On peut rappeler ici plusieurs exemples, dont celui de la Directive $97 / 7 / \mathrm{CE}$, ou le $17^{\mathrm{em}}$. considérant affirme que les principes établis par les articles 8 et 10 de la Convention européenne de sauvegarde des droits de l'homme et des libertés fondamentales du 4 novembre 1950, fondent la reconnaissance au consommateur d'un drott à la protection de la vie privée, notamment en ce qui concerne la tranquillité à l'égard de certaines techniques de communication particulièrement envahissantes. En conséquence, il y a lieu de préciser les limites spécifiques à l'usage de pareilles techniques. Parmi les points les plus importants du texte de cette Directive qui montrent ses sources comparatistes, il convient de signaler le droit à t'information, base de toutes les affaires liées à la consommation, et qui doit être le poinr de départ de tout achat. Si ce droit à l'information est fondamental dans n'importe quel achat, il l'est davantage quand il s'agit de contrats à distance. Inspiré par le droit allemand, qui impose aux contractants un comportement loyal et de bonne foi (les usages de commerce), les rédacteurs de cette Directive $97 / 7$ ont imposé aux fournisseurs un devoir de foumir, en temps utile, des informations préalables avant la conclusion de tour contrat à distance. I'information a pour but de rendre le consentement de l'acquéreur plus éclairé et, en conséquence, d'assurer l'équilibre contractuel, ainsi que l'égalité entre les parties.

En outre, le législateur communautaire peut trouver une solution inspirée des droits nationaux, mais qui les dépasse. Dans ce cas là, la solution devient originale, autonome. 